\title{
BUSINESS MODELS AND INFORMATION SYSTEMS FOR SUSTAINABLE DEVELOPMENT
}

\author{
Marten van Sinderen \\ University of Twente, Drienerlolaan 5, Enschede, The Netherlands \\ m.j.vansinderen@utwente.nl
}

\begin{abstract}
Keywords: Business models, Information systems, Goal modelling, Value modelling, Model-driven development, Sustainable development.

Abstract: Businesses are expected to explore market opportunities in the area of sustainable development, thus contributing to finding solutions aiming at sustainable quality of life. This will require adaptation and innovation of business models and information systems, with challenges of particular interest to the business modeling and software design community. This paper briefly discusses two relevant topics in this respect, namely (i) goal and value modeling, and (ii) model-driven development. We mention existing work that can be taken as a starting point for addressing sustainability issues, and we make some observations that may be taken into account when extending existing work.
\end{abstract}

\section{INTRODUCTION}

The IT-driven globalization has changed business and its underlying economic concepts (Friedman 2007). Also, people start to realize that consumption, financial profits and material ownership are not always positively correlated to well-being. A new paradigm for business and economics is needed, one which emphasizes sustainable development, where ecological, social and psychological dimensions of economic activity are recognized (Tideman 2005). This new paradigm will give quality of life a more central role. Although measurable indicators for sustainable development and quality of life are hard to define, business organizations will play an important role in shaping and testing it in practice. While business may have significantly contributed to the problem, companies to date are definitely considering sustainability issues, be it for reasons of governmental regulation, public image or genuine concern.

Thus, business organizations will explore market opportunities and thus exert activities that are potential solutions to the challenges of sustainable development, including reducing poverty, enhancing livelihoods, protecting ecosystems and tackling climate change (Wilson 2009). They have to invest in and rely on business models and information systems to be able to rationally and effectively address these challenges.
Business models specify how companies achieve their goals and realize a value proposition to their customers. Sustainable development at this level implies that not only economical aspects are taken into consideration when designing business processes, but social and environmental aspects as well. At the technical level, information systems (partially) automate the business processes. The design of information systems for sustainability should be optimized not only for delivering economic value, but also for providing social and environmental benefits. Figure 1 shows the role of business models and information systems in achieving a sustainable and balanced trade-off between economic, social and environmental aspects, both for individual business organizations as well as for business networks in which multiple business organizations collaborate.

Examples of areas where business models and information systems can be applied for sustainable development include smart energy grids (Ipakchi 2009, Warmer 2009), energy-efficient buildings and spaces for public use (Sinderen 2010, Teherian 20), energy-positive neighborhoods (Lopez 2011), and low carbon mobility and freight transport (Banister 2007, Steenwijk 2011). 


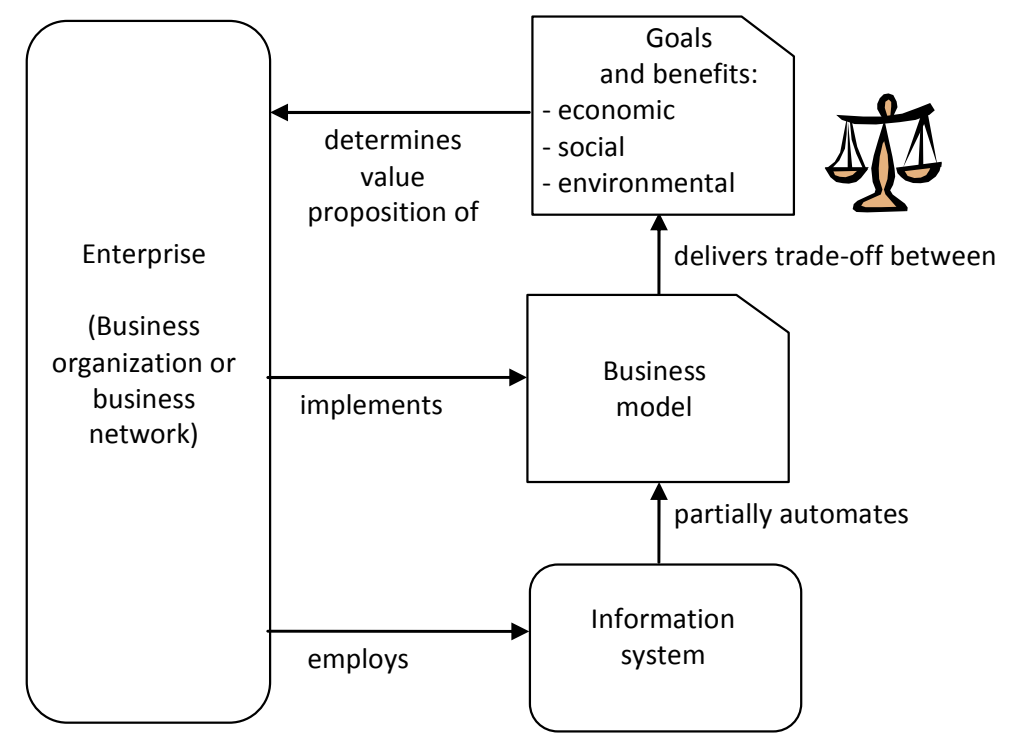

Figure 1: Role of business models and information systems in sustainability development.

We identify two important challenges related to sustainable development, which are of particular interest to the business modeling and software design community:

1. Goal and value modeling;

2. Model-driven development.

In the remaining of this paper we briefly explore these two topics. We mention existing work that can be taken as a starting point for addressing sustainability issues, and we make some observations that may be taken into account when extending existing work.

\section{GOAL AND VALUE MODELING}

Business models should be able to represent and enable reasoning about the goals and benefits of sustainable development. Trade-offs between the economic, social and environmental aspects of sustainable development goals need to be addressed (Wilson 2009). Goals of an enterprise lead to a value proposition to the enterprise's customers, which is expected to induce value exchanges between the enterprise and its customers. Value in this context can be anything worth in usefulness or importance to the stakeholders, ranging from money, goods, services (economic benefits), and equality, justice, liberty (social benefits), to a balanced ecosystem and stable climate (environmental benefits).

If the enterprise is a business network in which business partners collaborate, then the business partners have mutual value propositions and value exchanges between each other, in addition to value propositions to and value exchanges with customers. Assuming that all partners are essential for the collaboration, the success of the collaboration will depend on the achievement of a net value (profit or other benefit) for each of the partners. Also, in general, partners want a fair distribution of the overall net value of the collaboration, i.e., no partner should have disproportionate benefits considering his efforts spent and risks taken.

Enterprises are complex systems, hence creating models of such systems in order to understand and analyze their properties normally requires a structured, or multi-viewpoint, approach. Sustainability is one of these properties, which, as we have seen, can be related to multifaceted goals and values. Several techniques exist for goal and value modeling. A well-known approach to problem-oriented goal modeling is goal oriented requirements engineering (GORE), with techniques such as $i^{*}$ (Yu 1997) and KAOS (Dardenne 1993). A more recent development is ARMOR (Quartel 2009), which has the important benefit of being aligned with a standard enterprise modeling language, namely ArchiMate (TOG 2009). The most popular technique for value modeling is e3value (Gordijn 2002). The combined use of goal and value models has been explored in several works, including (Gordijn 2006, Mantovaneli 2009). However, none of the approaches and techniques explicitly addresses sustainability, and how economic, social and environmental aspects can be traded and balanced to allow sustainable development. 
Therefore, an important challenge is to extend business modeling, possibly based on current goal and value modeling techniques, but specifically targeted to sustainability. Such extensions may take account of the following observations:

- Trust is an important lubricant for business collaboration. Without a sufficient level of trust among potential partners, collaboration will not take place. Moreover, discovery of misplaced trust in a partner will lead to exclusion of that partner, and possibly to disintegration of the collaboration if the partner cannot be replaced. Initial work on trust and value modeling is reported in (Fatemi 2011a). We expect that a business organization can procreate trust from its potential business partners if it shows to respect or embrace similar values, particularly with respect to social and environmental benefits.

- Local communities maybe easier to involve in building partnerships than remote communities. Availability of background knowledge and trust recommendations plays a role in this, as well as similarity in culture and mutual understanding of values. Moreover, elimination of travel and transport may contribute to reduction of environmental impact.

- Personalization of business offerings to customers is an important factor of reinforcing customer relationships (Marca 2011). Customers are more interested if they feel that their needs and beliefs are taken seriously and if they can influence the value objects in the value exchanges with business organizations. In general, business organizations that are context-aware, i.e., aware of their customers' context but also of their operational context, may be able to exploit this knowledge to save energy and limit environmental impact.

\section{MODEL-DRIVEN DEVELOPMENT}

As mentioned earlier, at the technical level, information systems (partially) automate the processes and activities as described or constrained by business models. An information system will only be effective if it correctly implements the business models. Furthermore, maintenance of the information system will only be viable if properties expressed by the business models can be traced to capabilities of information system components. Changes to business models, e.g., triggered by market demands, can then be addressed at the technical level by adapting or replacing the components that support the affected business properties.

Model-driven development, based on the modeldriven architecture initiative of OMG (OMG 2003), is a widely accepted approach to manage system and software complexity and to achieve and maintain alignment between business level models and technology level models (Sinderen 2009). In particular, model-driven development organizes and relates the modeling space using a classification of models that offer different abstractions (Frankel 2003). These abstractions cover enterprise design and business network design together with the development of supporting software solutions. A key technical feature of model-driven development is the use of model transformations to (semi-) automatically derive 'target' models from 'source' models, where target models are typically of a lower level of abstraction than source models.

The challenge that is posed related to modeldriven development is then: can we propose model transformations that yield models of information systems to support sustainability, given one or more source business models. Given the complexity of enterprise systems, we cannot expect that a single transformation can be used to bridge between the business level and the information system level. Several approaches exist that consider transformations starting from either goal or value models. For example, (Mantovaneli 2009) explores the relations between value (e3value) models and goal (ARMOR) models; (Bandara 2004) presents an approach that applies goal decomposition to achieve policy refinement, using UML as high-level notation for both goals and policies; and (Fatemi 2011b) describes a transformation from a value (e3value) model to a coordination process (BPMN) model using the Groove transformation tool. The latter transformation result could be further exploited when targeting service-oriented information systems. For example, (Khadka 2011) proposes a transformation from a (CDL) model of an abstract coordination process between services to a concrete (BPEL) orchestration of services using the Atlas Transformation Language.

Many more transformation approaches exist, addressing a variety of modeling languages and employing various transformation techniques and tools. It is worthwhile to investigate whether existing approaches can be extended such that sustainability requirements can be preserved and sustainability solutions can be optimized. The 
following observations may be taken into account when developing model-driven sustainability:

- Reference models can be useful to guide the transformation process. Transformations from a higher to a lower abstraction level allow many (in principle, an infinite number of) possible target models given a single source model. This is because there are many ways to add lower level details that do not contradict the higher level properties represented by the source model. It may be difficult to know and formalize in advance the stakeholders' considerations that would allow automated selection of the 'best' solution. Hence, such situations would require frequent interaction with and input from a designer acting on behalf of the stakeholders. A well-founded and proven architectural model to which any target model must comply can limit the number of possible choices considerably, thus reducing designer interaction. For instance, (Balmus 2011) proposes a trade promotion management reference architecture that can be used to guide transformations of marketing business models.

- Monitoring and evaluation of sustainability parameters should be anticipated in business models, and should be implementation as far as possible in the supporting information systems. Alencar Silva (2011) presents an initial framework for configuring monitoring strategies from business requirements. A more elaborate framework is proposed in (Wetzstein 2011), which allows business analysts to determine how KPIs for business process performance depend on lower level process metrics and quality of service (QoS) characteristics of the information system infrastructure.

- Additional concepts may be required in languages, both at the business and technical level, to express properties relevant for sustainability development. These concepts should have a careful definition of semantics, in order to be useful in automated transformation approaches. This is especially true at the business level where sustainability sometimes deals subjective aspects of the enterprise. Moreover, enterprises generally have several stakeholders, involve collaboration between several business organizations, and require the integration of several architectural domains. The lack of a common interpretation of modeling concepts would inhibit model-driven development. The use of ontologybased semantics for concepts has been proposed as a viable approach to address this issue (Azevedo 2011). This work makes use of the Unified Foundational Ontology (UFO) as semantic foundation to evaluate, re-design and integrate models of conceptual modeling languages. Importantly for our purpose, UFO covers social and intentional phenomena, which need to be modeled when addressing sustainability development.

\section{CONCLUSIONS}

Traditional business models and information systems must be adapted and innovated in order to fit the new paradigm of sustainable development. Particular challenges for the business modeling and software design community are in the areas of goal and value modeling and model-driven development. We discussed the relationship between multifaceted (i.e., economic, social, environmental) goals and value propositions of an enterprise as a single business organization and as a business network. Trust, local communities and personalization present important boundary conditions for successful business models, and thus have to be explicitly considered in goal and value modeling. Modeldriven development can be used to achieve and maintain alignment between the business level models and technology level enterprise models. Particularly, automated model transformations are useful tools to cope with the dynamicity of business constellations and markets. However, current approaches in this area do not explicitly address sustainability. Extensions should consider the use of reference models (especially those that address sustainability), the need for monitoring and evaluation of sustainability parameters, and the definition of additional concepts with ontologybased semantics (using a foundational ontology).

\section{REFERENCES}

Friedman, T. (2007). The world is flat - The globalized world in the twenty-first century. Penguin Books.

Tideman, S. (2005). Mind over matter: towards a new paradigm for business and economics. In: Proceedings of the 2nd International Conference on Gross National Happiness - Rethinking Development - Local Pathways to Global Wellbeing.

Wilson, E., J. MacGregor, D. MacQueen, S. Vermeulen, B. Vorley, L. Zarsky (2009). Business models for sustainable development - Innovating for environment and society. International Institute for Environment and Development (IIED), Briefing, May 2009.

Ipakchi, A., F. Albuyeh (2009). Grid of the future. IEEE Power and Energy Magazine, Vol. 7, No. 2, pp. 52-62.

Warmer, C., K. Kok, et al. (2009). Web services for integration of smart houses in the smart grid. In: 
Proceedings The Road to an Interoperable Grid, GridInterop Forum, pp. 207-211.

Sinderen, M. van, M. Warnier, B. Shishkov (2010). Service-oriented architecture for household energy management. In: Proceedings of the 4th International Workshop on Enterprise Systems and Technology (IWEST 2010), SciTePress, pp. 52-69.

Taherian, S., M. Pias, G. Coulouris, J. Crowcroft (2010). Profiling energy use in households and office spaces. In: Proceedings of the 1st International Conference on Energy-Efficient Computing and Networking (eEnergy 2010), ACM, pp. 21-30.

López, G., P. Moura, J. Ignacio Moreno, A. de Almeida (2011). ENERsip: M2M-based platform to enable energy efficiency within energy-positive neighbourhoods. In: Proceedings of the IEEE INFOCOM Workshop on Machine-to-Machine Communications and Networking (M2MCN 2011), IEEE, pp. 217-222.

Banister, D. (2007). The sustainable mobility paradigm. Transport Policy, Vol. 15, No. 2, pp. 73-80

Steenwijk, M. (2011). A reference architecture for fuelbased carbon management information systems in the logistics industry. MSc thesis, University of Twente.

Yu, E. (1997). Towards modelling and reasoning support for early-phase requirements engineering. In: Proceedings of the 3rd IEEE International Symposium on Requirements Engineering (RE 1997), IEEE, pp. 226-235.

Dardenne, A., A. van Lamsweerde, S. Fickas (1993). Goal-directed requirements acquisition. Science of Computer Programming. Vol. 20, pp. 3-50.

Quartel, D.A.C., W. Engelsman, H. Jonkers, M. van Sinderen, (2009) A goal-oriented requirements modelling language for enterprise architecture. In: Proceedings of the 13th IEEE International EDOC Enterprise Computing Conference (EDOC 2009), IEEE Computer Society, pp. 3-13.

The Open Group (2009). ArchiMate 1.0 Specification. http://www.opengroup.org/archimate.

Gordijn, J., H. Akkermans (2002). Value based requirements engineering: exploring innovative ecommerce ideas. Requirements Engineering Journal, Vol. 8, pp. 114-134.

Gordijn, J., M. Petit, R.J. Wieringa, R.J. (2006). Understanding business strategies of networked value constellations using goal- and value modeling. In: Proceedings of the 14th IEEE International Requirements Engineering Conference (RE 2006), IEEE computer Society, pp. 129-138.

Mantovaneli Pessoa, R., M. van Sinderen, D.A.C. Quartel (2009). Towards requirements elicitation in serviceoriented business networks using value and goal modelling. In: Proceedings of the 4th International Conference on Software and Data Technologies (ICSOFT 2009), INSTICC, pp. 392-399.

Fatemi, H., M. van Sinderen, R. Wieringa (2011a). Trust and value webs. In: Proceedings of the 15th IEEE International EDOC Enterprise Computing Conference
(EDOC 2011), IEEE Computer Society, 10 pp. (to appear).

Marca, D., R. Bulander, C. Kruslin, B. Shishkov, M. van Sinderen (2011). E-business challenges and directions. In: Revised Selected Papers of the 7th International Joint Conference on e-Business and Telecommunications (ICETE 2010), Springer, 33 pp. (to appear).

Open Management Group (2003). MDA Guide Version 1.0.1. Document No. omg/2003-06-01, 62 pp. http://www.omg.org/mda.

Sinderen, M. van, M. Spies (2009). Towards model-driven service-oriented enterprise computing. Enterprise Information Systems, Vol. 3, No. 3, pp. 211-217.

Frankel, D.S. (2003). Model driven architecture: applying MDA to enterprise computing. JohnWiley and Sons.

Bandara, A.K., E.C. Lupu, J. Moffett, A. Russo (2004). A goal-based approach to policy refinement. In: Proceedings of the 5th Workshop on Policies for Distributed Systems and Networks (Policy 2004), IEEE Computer Society, 11 pp.

Fatemi, H., M. van Sinderen, R. Wieringa (2011b). E3value to BPMN model transformation. In: Proceedings of the 12th IFIP Working Conference on Virtual Enterprises (PRO-VE 2011), Springer, 8 pp. (to appear).

Khadka, R., B. Sapkota, L. Ferreira Pires, M. van Sinderen, S. Jansen (2011). Model-driven development of service compositions for enterprise interoperability. In: Proceedings of the 3rd International IFIP Working Conference on Enterprise Interoperability (IWEI 2011), LNCS 76, Springer, pp. 177-190.

Bulmus, A.B. (2011). Reference system architecture for trade promotion management leveraging business intelligence technologies and decision support systems. MSc thesis, University of Twente.

Alencar Silva, P. de, H. Weigand (2011). On the move to business-driven alignment of service monitoring requirements. In: Proceedings of the 3rd International IFIP Working Conference on Enterprise Interoperability (IWEI 2011). LNBIP 76, Springer, pp. 103-117.

Wetzstein, B., P. Leitner, F. Rosenberg, S. Dustdar, F. Leymann (2011). Identifying influential factors of business process performance using dependency analysis. Enterprise Information Systems, Vol. 5, No. 1, pp. 79-98.

Azevedo, C.L.B., J.P. A. Almeida, M. van Sinderen, D. Quartel, G. Guizzardi (2011). An ontology-based semantics for the motivation extension to ArchiMate. In: Proceedings of the 15th IEEE International EDOC Enterprise Computing Conference (EDOC 2011), IEEE Computer Society, 10 pp. (to appear). 